\title{
Interlaminar crack initiation and growth under modes I and II in a carbon-fibre epoxy composite subjected to fatigue
}

\author{
A. Argüelles ${ }^{1}$, J. Viña ${ }^{2}$, A. F. Canteli ${ }^{1} \&$ J. Bonhomme ${ }^{3}$ \\ ${ }^{I}$ Department of Construction and Manufacturing Engineering, \\ University of Oviedo, 33203 Gijón, Spain \\ ${ }^{2}$ Department of Materials Science and Metallurgical Engineering, \\ University of Oviedo, 33203 Gijón, Spain \\ ${ }^{3}$ ITMA Foundation, Technological Centre of Non Metallic Materials, \\ 33428 Llanera, Spain
}

\begin{abstract}
In this paper, interlaminar crack initiation and propagation under modes I and II dynamic loading of a composite material, with epoxy matrix and unidirectional carbon fibres were evaluated. Delamination commenced in mode I employing the DCB (Double Cantilever Beam) test. In mode II, the ENF (End Notched Flexure) test was used. The fracture toughness in mode I was obtained using the methods of the ASTM D5528 Standard, while in mode II, the methods were applied in accordance with the ESIS (European Structural Integrity Society) Protocol. Employing this experimental program, the fatigue curves $(\Delta \mathrm{G}, \mathrm{N})$ and growth rate curves $(\Delta \mathrm{G}, \mathrm{da} / \mathrm{dN})$ in both fracture modes were determined for an asymmetry ratio of $\mathrm{R}=0,2$. The influence of the manufacturing process of the material on its behaviour with respect to crack growth onset may be deduced from the experimental results. Moreover, as the crack growth rate decreases for large crack lengths, crack growth may even cease if the critical fracture energy does not increase above the values obtained in the static characterization of the material.
\end{abstract}

Keywords: delamination, fatigue, fracture, crack growth. 


\section{Introduction}

The high specific strength and stiffness of composite materials have been of great importance in their use in structures where weight is a critical variable. However, composites made of laminates exhibit a worrying susceptibility towards the appearance and growth of cracks between the layers. This phenomenon, known as delamination, represents one of the most life-limiting failure modes of laminated composites. Delamination can be caused either by defects induced during the manufacturing process or generated in structural elements during the service life of the material due to interlaminar stresses that can lead to the commencement of delamination. Although several decades have elapsed since the recognition of the importance of interlaminar failure $[1,2]$, it stills remains a determining factor limiting the use of structural elements made of laminated composites [3]. In recent years, a number of research projects have been conducted that focus on the determination of the characteristics of interlaminar failure under static loading [4-6]. However, new developments, predominantly in the aerospace field, compel us to complete the information on the behaviour of these materials with reference to delamination under varying loading conditions [7-12]. In this study, the initiation and propagation of interlaminar cracks in mode I and II fracture and dynamic loading were analyzed in a composite material made of an epoxy matrix reinforced with unidirectional carbon fibres. With regard to these tests, while the mode $\mathrm{O}$ test is clearly defined and has been presented as an international standard, the mode II test gives rise to a certain degree of controversy and is currently under development by different international research groups.

\section{Experimental procedure}

\subsection{Materials and specimens}

The composite material employed in this research program was manufactured from Hexcel 8552 epoxy resin prepregs reinforced with unidirectional AS4 carbon fibre, with commercial denomination Hexcel AS4/8552 RC34 AW196. The mechanical properties were obtained according to ASTM D3039 Standard for tension and according to ASTM D3518 Standard for shear, providing the results in Table 1 .

Table 1: $\quad$ Mechanical properties of the material.

\begin{tabular}{|c|c|c|c|c|c|}
\hline \multicolumn{2}{|c|}{ Elastic Moduli } & \multicolumn{2}{|c|}{ Tensile Strength } & $\begin{array}{c}\text { Shear } \\
\text { Modulus }\end{array}$ & $\begin{array}{c}\text { Shear } \\
\text { Strength }\end{array}$ \\
\hline $\begin{array}{c}\mathrm{E}_{11} \\
(\mathrm{GPa})\end{array}$ & $\begin{array}{c}\mathrm{E}_{22} \\
(\mathrm{GPa})\end{array}$ & $\begin{array}{c}\sigma_{11} \\
(\mathrm{MPa})\end{array}$ & $\begin{array}{c}\sigma_{22} \\
(\mathrm{MPa})\end{array}$ & $\begin{array}{c}\mathrm{G}_{12} \\
(\mathrm{GPa})\end{array}$ & $\begin{array}{c}\tau_{\max } \\
(\mathrm{MPa})\end{array}$ \\
\hline 144 & 10,6 & 1703 & 30,8 & 5,36 & 67,7 \\
\hline C.V.:7,8\% & C.V.:5,9\% & C.V.:9,2\% & C.V.:11\% & C.V.:12\% & C.V.:1,6\% \\
\hline
\end{tabular}


The same type of specimen was used for the delamination tests in modes I and II. Figure 1 shows the geometry of these specimens in accordance with ASTM D5528 [13] and ESIS Protocol [14]. The specimens are rectangular, uniform in width and height, and the configuration is $\left[0_{16} /\right.$ insert $\left./ 0_{16}\right]$ with a nonadhesive insert placed midplane to provoke the initial delamination.

The specimens were previously dried, thus avoiding any influence of ambient conditions on the mechanical tests.

(11- direction of fibres, 22- perpendicular to fibres, C.V.: coefficient of variation)

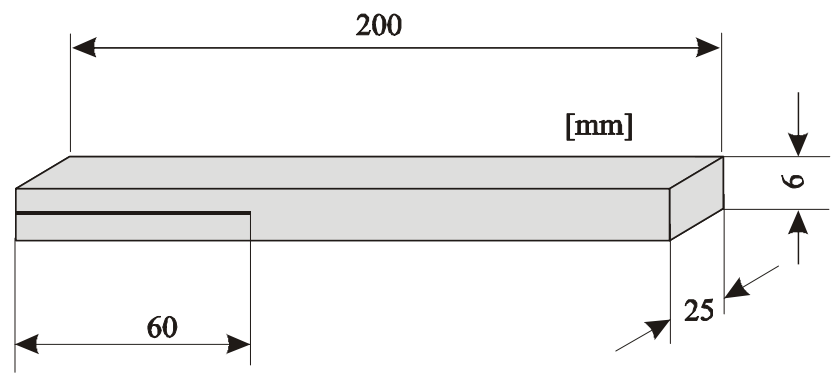

Figure 1: Geometry of the specimens.

\subsection{Static and fatigue characterization}

All the tests performed to characterize the material in modes I and II under static and cycling loading were carried out in a servo-hydraulic MTS testing machine.

\subsubsection{Static fracture toughness in mode I}

To determine the static fracture toughness in Mode I, three methods were applied in accordance with the ASTM D5528 Standard: the Modified Beam Method (MBT), the Compliance Calibration Method (CC) and the Modified Compliance Calibration Method (MCC). A deformation rate of $0.5 \mathrm{~mm} / \mathrm{min}$ was applied throughout the tests.

The experimental results obtained for the static fracture toughness $\mathrm{G}_{\text {Ic }}$ according to the three methods are summarized in Table 2. As the method that presents least deviation is MBT, then this method will be used for obtaining the values of the dynamic tests.

Table 2: $\quad$ Fracture toughness in mode I.

\begin{tabular}{|l|c|c|c|}
\hline & $\begin{array}{c}\mathrm{G}_{\mathrm{Ic}}\left(\mathrm{J} / \mathrm{m}^{2}\right) \\
(\mathrm{MBT})\end{array}$ & $\begin{array}{c}\mathrm{G}_{\mathrm{Ic}}\left(\mathrm{J} / \mathrm{m}^{2}\right) \\
(\mathrm{CC})\end{array}$ & $\begin{array}{c}\mathrm{G}_{\mathrm{Ic}}\left(\mathrm{J} / \mathrm{m}^{2}\right) \\
(\mathrm{MCC})\end{array}$ \\
\hline Average & 302.105 & 319.531 & 298.082 \\
\hline Typical deviation & 29.155 & 39.382 & 35.666 \\
\hline C. V. (\%) & 9.650 & 12.325 & 11.965 \\
\hline
\end{tabular}




\subsubsection{Static fracture toughness in mode II}

To determine the static fracture toughness in mode II, four methods were applied in accordance with the ESIS Protocol [14]: Experimental Compliance Calibration (CE), Direct Beam Theory ( $\left.\mathrm{BT}_{\text {direct }}\right)$ and the Area Method (A). The experimental results obtained for the static fracture toughness $\mathrm{G}_{\text {IIc }}$ according to the four methods are summarized in Table 3 . In this case, the method with least deviation is $\mathrm{BT}_{\text {direct}}$, which will be used for obtaining the values of the dynamic tests.

Table 3: $\quad$ Fracture toughness in mode II.

\begin{tabular}{|c|c|c|c|c|}
\hline & $\begin{array}{c}\mathrm{G}_{\text {IIc }}\left(\mathrm{J} / \mathrm{m}^{2}\right) \\
(\mathrm{CE})\end{array}$ & $\begin{array}{c}\mathrm{G}_{\text {IIc }}\left(\mathrm{J} / \mathrm{m}^{2}\right) \\
\left(\mathrm{BT}_{\text {direct }}\right)\end{array}$ & $\begin{array}{c}\mathrm{G}_{\text {IIc }}\left(\mathrm{J} / \mathrm{m}^{2}\right) \\
\left(\mathrm{BT}_{\mathrm{E}}\right)\end{array}$ & $\begin{array}{c}\mathrm{G}_{\text {IIc }}\left(\mathrm{J} / \mathrm{m}^{2}\right) \\
(\mathrm{A})\end{array}$ \\
\hline Average & 1062.080 & 1125.006 & 1146.378 & 1109.266 \\
\hline Typical deviation & 199.811 & 160.403 & 205.929 & 170.953 \\
\hline C.V. $(\%)$ & 18.813 & 14.258 & 17.963 & 15.411 \\
\hline
\end{tabular}

\subsubsection{Fatigue characterization}

The aim of the fatigue tests was to provide information on the material to be used in the fatigue life prediction of laminates. This objective was twofold:

a) To ascertain the number of cycles needed to initiate delamination for a given energy release rate, i.e., to determine the fatigue curves $\Delta \mathrm{G}-\mathrm{N}$ for an asymmetry ratio $\mathrm{R}=0.2$.

b) To characterize the propagation phase by means of the crack growth curve $\mathrm{da} / \mathrm{dN}-\Delta \mathrm{G}$.

The onset of delamination growth was decided by visual observation, i.e., the number of crack initiation cycles was the number of cycles from the beginning of the test to the visual observation of a crack at the end of the insert $[15,16]$. These results are very similar to those obtained from the conventional fatigue test. The crack growth rates (da/dN- $\Delta \mathrm{G}$ curves) were determined for different fractions of $\mathrm{G}_{\text {crit. }}$. Crack length values were read by means of a $100 \mathrm{x}$ travelling microscope located at one side of the specimens.

Although the test is difficult and laborious under these conditions, the methodology is sufficiently reliable and the information obtained valid, even taking into account the different growth of the crack in the width of the specimen. As in the crack initiation tests, the asymmetry coefficient was 0.2 .

The test was stopped every 1000 cycles in order to determine the crack length and consequently the crack growth rate by optical observation without removing the specimen from the fixture. The energy release rate for the last 1000 cycles was evaluated using the mean values of the crack length and the maximum and minimum loads. A frequency of $3 \mathrm{~Hz}$ was applied throughout the tests.

\section{Experimental results}

The experimental results obtained from the tests are presented below. 


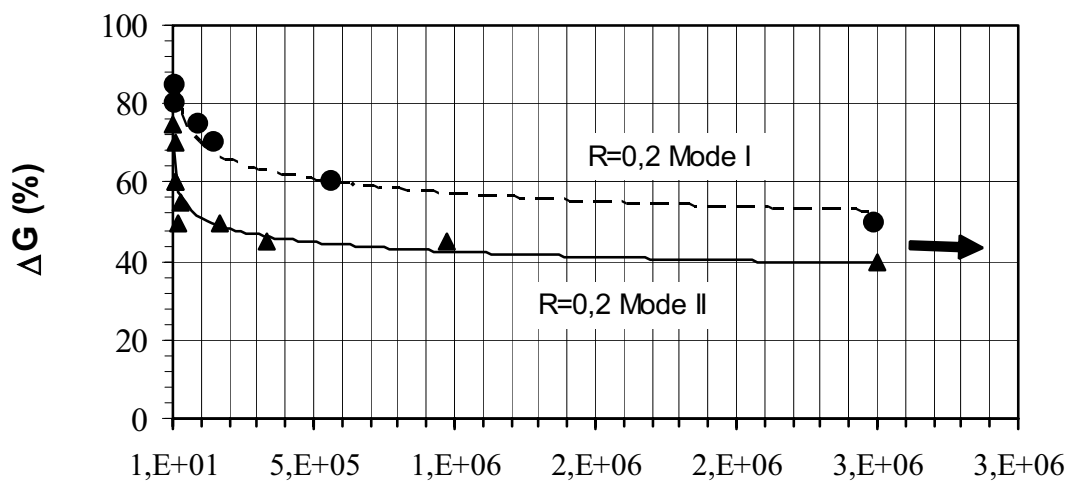

\section{Number of cycles}

Figure 2: $\quad$ Fatigue curves of the material.

\subsection{Onset of delamination}

Figure 2 represents the curves characterizing the fatigue behaviour of the material (crack growth onset) for the two modes of fracture tested.

The values of the critical energy resulting from the tests are represented as a percentage. A fatigue limit, discretionally taken as the fatigue strength corresponding to 3 million cycles, i.e., the limit number of cycles, corresponds to approximately $50 \%$ of the critical fracture energy in mode I calculated from the static tests according to the Modified Beam Method $\left(\Delta \mathrm{G}=120.8 \mathrm{~J} / \mathrm{m}^{2}\right)$ and $40 \%$ in mode II according to the Direct Beam Theory $\left(\Delta \mathrm{G}=360 \mathrm{~J} / \mathrm{m}^{2}\right)$.

The pronounced scatter observed in the results of the number of cycles elapsed until crack initiation could presumably be assigned to the strip of resin accumulated at the crack front as a result of the manufacturing process. Also, the difficulty of the exact determination of the onset of the crack must be considered because this onset has been detected visually.

\subsection{Delamination crack rate}

Figures 3 and 4 show the crack growth rate versus crack length for the test material for different loading levels and both fracture modes.

Figure 3 presents the behaviour of the material for loading levels corresponding to 80 and $75 \%$ of the critical delamination energy in mode I. The mean crack growth rates obtained were $1.0063 \cdot 10^{-4} \mathrm{~mm} / \mathrm{cycle}$ for $80 \%$ and $2.82756 \cdot 10^{-5} \mathrm{~mm} /$ cycle for $70 \%$.

Figure 4 presents the behaviour of the material for loading levels corresponding to $60,55,50$ and $45 \%$ of the critical delamination energy in mode II. The mean crack growth rates obtained were $1.1 \cdot 10^{-4}, 4.59 \cdot 10^{-4}, 4.15 \cdot 10^{-4}$ and $9.97 \cdot 10^{-4} \mathrm{~mm} /$ cycles for loading levels corresponding to $60,55,50$ and $45 \%$, respectively. 


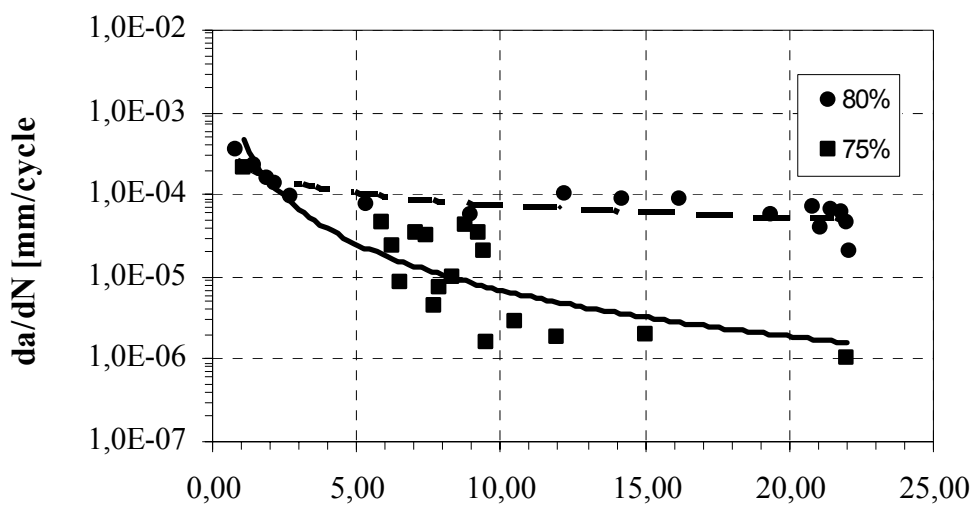

Crack length $[\mathrm{mm}]$

Figure 3: $\quad$ Crack growth rate versus crack length for mode I.

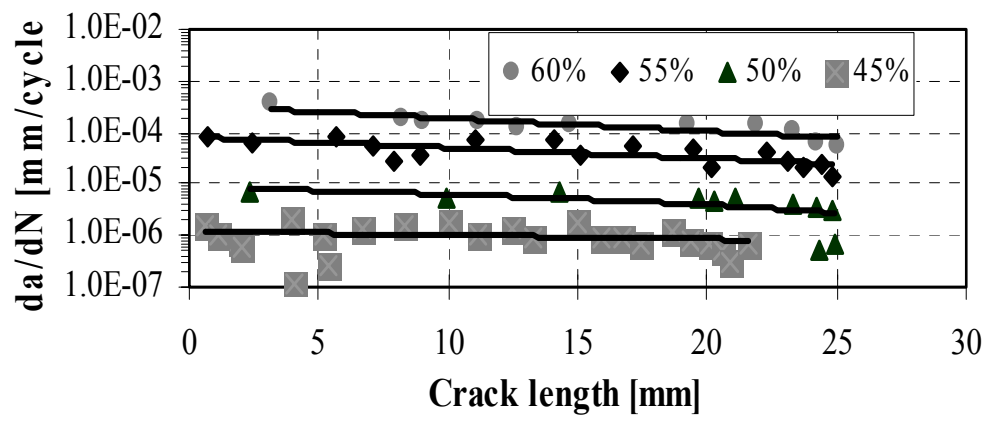

Figure 4: $\quad$ Crack growth rate versus crack length for mode II.

In both failure modes, the growth rate decreases with increasing crack length. Figure 5 represents both fracture modes on the same graph for the same crack length.

Although the overall behaviour is similar in both cases, there are several differences. While the crack growth rate in mode II presents the same trend for all the loading levels, behaviour in mode I is different. At the high loading level, behaviour is similar to mode II. For a loading level of $75 \%$, the growth rate decreases strongly with increasing crack length. The reason could lie in the formation of fibre bridging, which decreases the rate of the process.

Figure 6 represents the evolution of the crack growth rate in fatigue versus the maximum normalized delamination energy $\left(\mathrm{G}_{\max } / \mathrm{G}_{\mathrm{c}}\right)$ for both fracture modes. This energy was calculated using the Modified Beam Method (MBT) for mode I and the Direct Beam Theory $\left(\mathrm{BT}_{\text {direct }}\right)$ for mode II. 


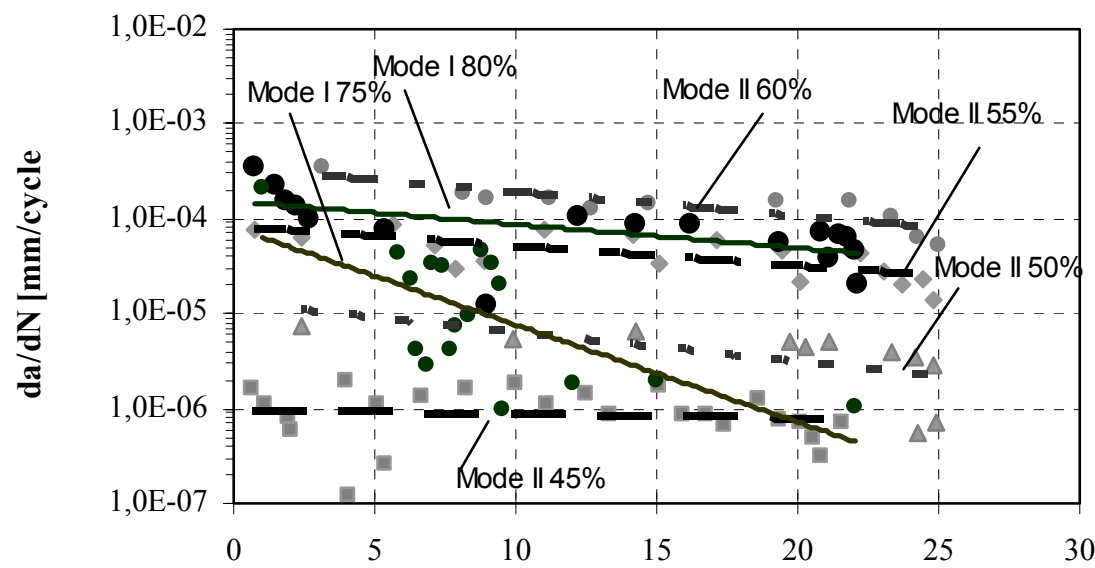

\section{Crack length $[\mathrm{mm}]$}

Figure 5: $\quad$ Crack growth rate versus length for modes I and II.

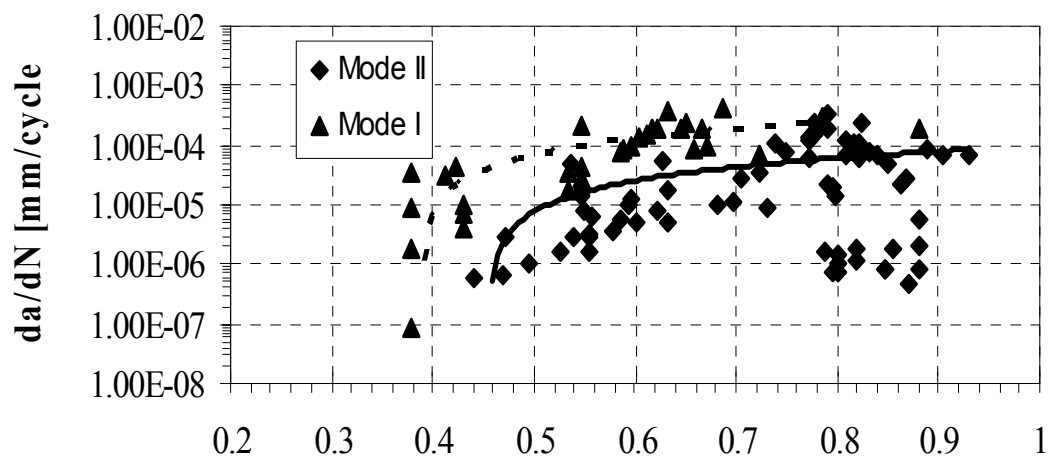

\section{Gmax/Gc}

Figure 6: Crack growth rate versus maximum normalized delamination energy.

In this figure, the same trend can be observed in the growth rate, i.e., the higher values of the growth rate correspond to the higher increments in critical energy.

\section{Conclusions}

In this paper, certain parameters controlling the delamination process of a composite under modes I and II fatigue loading were experimentally investigated. The results may be summarized as follows: 
- As regards interlaminar crack initiation

The determination of the fatigue curves was strongly influenced by the manufacturing process of the laminate and the accumulation of resin at the crack front, which led to pronounced variability in the data. Accordingly, the study and interpretation of the influence of these parameters requires the testing of a large number of specimens. The fatigue curves present the normal shape, i.e., the number of cycles increases if the critical energy decreases.

As regards crack growth

Crack growth slows down with increasing crack lengths and can even stop progressing if the critical fracture energy supplied does not exceed the equivalent values calculated from the static characterization of the material, for both modes of fracture and for all tested stress levels. However, this phenomenon could be caused by the increasing flexibility of the specimens' lips under dynamic loading and by the presence of fibre bridging, which can use up a fraction of the applied energy to the specimen.

\section{References}

[1] O'Brien, T.K., Characterization of delamination onset and growth in a composite laminate. Damage in Composite Materials, ASTM STP 775, ed. K.L. Reifsnider, pp. 140-167, 1982.

[2] Standard tests for toughened resin composites. NASA RP 1092, 1983

[3] Paul, D., Kelly, L., Venkayya, V., Hess, T., Evolution of U.S. military aircraft structures technology. Journal of Aircraft, 39 (1), pp. 18-29, 2002.

[4] Hojo, M., Matsuda, S., Tanaka, M., Göran Gustafson, C., Hahashi, R., Effect of stress ratio on near-threshold propagation of delamination fatigue cracks in unidirectional CFRP, Composites Science and Technology, 29, pp. 273-292, 1987.

[5] Hansen, U., Gillespie, J. W., Dependence of intralaminar fracture toughness on direction of crack propagation in unidirectional composites, Journal of Composites Technology and Research, 20, pp. 89-99, 1998.

[6] Brunner, A. J., Experimental aspects of Mode I and Mode II fracture toughness testing of fibre-reinforced polymer-matrix composites, Computer Methods Applied Mechanics Engineering, 185, pp. 161-172, 2000.

[7] Ashcroft, I. A., Hughes, D. J., Shaw, S. J., Mode I fracture of epoxy bonded composite joints: 1. Quasi-static loading, International Journal of Adhesion and Adhesives, 21, pp. 87-99, 2001.

[8] Hojo, M., Ando, T., Tanaka, M., Adachi, T., Ochiai, S., Endo, Y., Modes I and II interlaminar fracture toughness and fatigue delamination of CF/epoxy laminates with self-same epoxy interleaf. International Journal of Fatigue, 28, pp. 1154-1165, 2006.

[9] Shivakumar, K., Chen, H., Abali, F., Le, D., Davis, C., A total fatigue life model for mode I delaminated composite laminates, International Journal of Fatigue, 28, pp. 33-42, 2006.

[10] Mahdi, S., Kim, H. J., Gama, B.A., Yarlagadda, S., Gillespie, J.W., A comparison of oven-cured and induction-cured adhesively bonded 
composite joints, Journal of Composite Materials, 37 (6), pp. 519-542, 2003.

[11] Matsubara, G., Ono, H., Tanaka, K., Mode II fatigue crack growth from delamination in unidirectional tape and satin-woven fabric laminates of high strength GFRP, International Journal of Fatigue 28, pp. 1177-1186, 2006.

[12] Qiao, P., Hu, G., Mode II fatigue fracture of wood-composite bonded interfaces, Journal of Composite Materials, 38 (6), pp. 453-473, 2004.

[13] ASTM D 5528-01, Mode I interlaminar fracture toughness of unidirectional fiber-reinforced polymer matrix composites.

[14] ESIS Protocols for interlaminar fracture testing of composites.

[15] Hojo, M., Matsuda, S., Tanaka, M., Ochiai, S., Murakami, A., Mode I delamination fatigue properties of interlayer-toughened CF/epoxy laminates, Composites Science and Technology, 66, pp. 665-675, 2006.

[16] ASTM D 6117-97 Mode I fatigue delamination growth onset of unidirectional fiber-reinforced polymer matrix composites. 